\title{
7. CONSCIOUSNESS: A REAL MYSTERY
}

\section{https://doi.org/10.36592/9786587424903-07}

\section{Erhan Demircioğlu}

The question I will address in this paper is an old one, whether consciousness is a real mystery, a mystery about the fabric of our universe itself. This question is, as will become clear, intimately tied to the question why consciousness appears to be a real mystery. If consciousness is not a real mystery but only appears to be so (that is, if what I will call "appearance mysterianism" is true), as many philosophers tend to believe, then we face the question "-the illusion question" - why consciousness appears to be a real mystery when, in fact, it is not. Without an adequate answer to the illusion question, appearance mysterianism is undermined. And, as I will argue, the attempts currently available to answer the illusion question fail, which should rationally incline us away from appearance mysterianism and towards "de re mysterianism", the view that consciousness is a real mystery.

\section{Consciousness: A Curious Phenomenon}

I am a conscious body but the cup lying motionless on my desk is an unconscious (or, more adequately, non-conscious) thing. I have sensations, feelings, and thoughts; the cup has no sensations, feelings, or thoughts. This striking difference between me and the cup cries out for an explanation: what is it that explains why I am, but the cup is not, conscious? It seems that it must be the case that I am conscious in virtue of something, some property the body I am has. And, it must be the case that the cup is non-conscious in virtue of lacking something, some property it fails to have. It cannot be a brute, inexplicable fact that I am conscious but the cup is not. There must be something that explains why I am conscious and the cup is not; and, setting aside super-natural interventions (a practice that I will follow throughout the paper), the explanation must have to do with the body I am and the body the cup is. There must be a difference between my body and the cup, a difference that explains why I am conscious but the cup is not. 
The mind-body problem springs from the observation that there seems to be nothing in my body that might explain why I am conscious (or have conscious states). Surely, a certain part of me, my brain, is remarkably different from the parts of the cup, however you wish to carve it, and there are good reasons to think that my being conscious is intimately tied to my having a brain. Taking a strong hit on the head, I might go blind, lose the capacity to have olfactory experiences or, even more unfortunately, turn totally unconscious. This points to the fact that there is a correlation (or perhaps even a causal relation) between my being conscious and (some of) my brain states, viz. that my brain is, so to speak, the seat of my consciousness. Of course, however, my brain's being the seat of my consciousness does not explain why it is the seat of my consciousness (it does not explain itself!). Furthermore, and more significantly, the question of what it is in my body that explains why I am conscious can be reformulated, without losing its force, as the question of what it is about my brain that explains why my brain is the seat of my consciousness. There is a correlation between (some of) my brain states and my being conscious, while there is no correlation between any of the states of the parts of the cup and its being conscious (simply because the cup is not conscious). But why? There must be something about my brain in virtue of which it is the seat of my consciousness, what is it? The problem is that there seems to be nothing special, or at least special enough, about my brain that explains why it is the seat of my consciousness. At a micro-physical level, my brain is just a swarm of atoms organized in a certain way, and it is rather difficult to see how the organization of atoms can give rise to consciousness. At a macro-physical level, my brain is just a kind of meat, and it is again rather difficult to see how a kind of meat can be conscious. So, despite the uncontroversial fact that we can point at some differences, at both levels, between my brain and the cup, it does not seem that those differences are capable of explaining what it is about the former that makes me conscious and the latter that makes it non-conscious.

\section{De Re Mysterianism}

There is thus no question that consciousness appears mysterious. Does it 
follow that consciousness is mysterious? The answer is no. The possibility that there is something in my body that explains why I am conscious is compatible with the fact that it appears that there is nothing in my body that explains (or could explain) why I am conscious. Perhaps, it only appears to us (or maybe even to conceiving minds in general) that consciousness is mysterious, whereas it is in reality as mundane and unexceptional as other products of our bodies such as bile.

Let us call the thesis that consciousness is (and not merely appears to be) mysterious "de re mysterianism". A nice thing about de re mysterianism is that it provides a plausible and straightforward explanation of why consciousness seems mysterious: the answer is, according to de re mysterianism, that consciousness is mysterious. The real mysteriousness of consciousness, on this view, explains why it seems mysterious. The mysterious appearance, in this case, is simply a reflection of something real, the mysterious reality itself. However, despite this explanatory advantage, it is hard to believe that de re mysterianism is true because to believe that consciousness is mysterious (that is, to believe that there is no explanation of consciousness in terms of bodily features) is to believe that nature is miraculous (that there are things in nature that simply happen, without there being an explanation of their occurrence in terms of more fundamental things). Can we really believe, given our current understanding of how it actually works, that there are miracles in nature?

It is, to say the least, hard to believe that de re mysterianism is true; however, I will attempt to show in this paper that that is the conclusion towards which we are inescapably driven. My argument is, briefly put, that the alternative to de re mysterianism, which I will call "appearance mysterianism", fails to provide an adequate answer to a particular question, a question it needs to answer in order to be a viable option. And, it is the very failure of appearance mysterianism that should rationally move us towards de re mysterianism, the thesis that consciousness is a real mystery.

\section{Appearance Mysterianism}

Appearance mysterianism is the thesis that combines the de re nonmysteriousness of consciousness with the apparent mysteriousness of 
consciousness. Note that appearance mysterianism is a weak position: it simply recognizes that consciousness seems mysterious (that, it seems that there is no explanation of consciousness in terms of bodily features) and maintains that consciousness is not mysterious (that there is indeed an explanation of consciousness in physical terms). Anyone who satisfies these two conditions (and only these two conditions) is an appearance mysterian.

Appearance mysterianism faces a central challenge and needs to answer a central question. Let me first spell out the challenge. If it seems to us that there is nothing in our bodies that can be appealed to in the quest for an explanation of consciousness, as appearance mysterianism says it does, then what good reason do we have to think that there is something in them that can be appealed to in such a quest? Is not the failure to pin down that very thing, whatever it is, that explains consciousness, a reliable indicator that the insistence that the mystery of consciousness is merely apparent is bound to look, sooner or later, as an instance of unreasonable stubbornness? If the appearance of a mystery is a defeasibly good reason for its reality, as it seems to be, then unless the force of the appearance is defeated, the reasonable attitude seems to be to believe in the reality of the mystery. So, the challenge for appearance mysterianism is to reconcile its two defining conditions that pull in opposite directions. I dub this challenge the stability challenge.

Now, here is the question that appearance mysterianism must answer: why does consciousness seem mysterious, if it is not mysterious? Why does it seem to us that there is nothing in our bodies that can be appealed to in the quest for an explanation of consciousness, while there is in fact something in our bodies that suits the job? What is it that explains the illusion that the physical matter seems utterly incapable of making the existence and character of consciousness intelligible? The relief provided by appearance mysterianism that consciousness is not mysterious is immediately accompanied with the question of what it is that makes it appear mysterious. Let me call this question the illusion question.

A satisfactory response to the stability challenge requires that the appearance mysterian find a way to support the idea of the non-mysteriousness of consciousness while defeating the support the apparent mysteriousness of consciousness provides to the idea of mysteriousness of consciousness. A 
satisfactory response to the illusion question requires that the appearance mysterian explain the apparent mysteriousness of consciousness in a way that is consistent with the idea that it is not mysterious. The former involves the task of defeating the support the appearance provides to the idea of mysteriousness of consciousness, and the latter the task of explaining why there is such an appearance in the first place. Thus formulated, the challenge and the question are at least notionally different.

However, it is important to realize that a satisfactory response to the illusion question automatically qualifies as a proper part of a satisfactory response to the stability challenge. That is, if the apparent mysteriousness of consciousness is explained in a way that is consistent with the idea that it is not mysterious, then the support the apparent mysteriousness of consciousness provides to the idea of mysteriousness of consciousness is thereby defeated. This is because the support in question is plausibly conditional on there being no explanation of the appearance in question other than the explanation in terms of the real mystery of consciousness.

The kind of defeat that would be at work if the illusion question were answered is what is called "undercutting defeat." Here is a paradigmatic case of undercutting defeat. I enter my friend's reading room and have an experience as of seeing some red books. My experience provides support to the belief that there are red books in the room. However, my friend later informs me that the books in the room are intricately illuminated by red light. My friend's testimony is not evidence that there are no red books in the room; however, it still defeats the support my experience provides to the belief that there are red books. But why exactly is the belief defeated? A plausible answer is that, given my friend's testimony, there are now two different ways available to me in which my having an experience as of seeing some red books can be explained: one appeals to the books 'being red, and the other appeals to the lightning conditions. So, the support my experience at hand provides to the belief that there are red books is defeated by my friend's testimony because my friend's testimony provides an alternative explanation of why I have that very experience. In this case, the support my experience provides to the belief is undercut. It must be clear that analogous considerations apply to the consciousness case. If the apparent mysteriousness of consciousness can be explained in a way consistent with the idea 
that it is not mysterious, then the support the appearance in question provides to the idea of mysteriousness of consciousness is undercut.

It might be argued that even though a response to the illusion question automatically qualifies as a proper part of a response to the stability challenge, a response to the stability challenge does not require a response to the illusion question. This is because, it might be further maintained, a response to the stability challenge requires only that the support the apparent mysteriousness of consciousness provides to the idea of mysteriousness of consciousness be defeated. Moreover, since undercutting defeat, the kind of defeat that would be at work if the illusion question were answered, is only one of two main kinds of defeat (the other one being "rebutting defeat"), it might be claimed that the other kind of defeat might be attempted in the search for a response to the stability challenge. And, if the stability challenge can be met without answering the illusion question, then it might be argued that however interesting that question is and however preferable it would be to answer it, appearance mysterianism is not required to answer it in order to be a satisfactory position. And, this is because, it might be thought, once the support the apparent mysteriousness of consciousness provides to the mysteriousness of consciousness is defeated, there is nothing more about that appearance that makes it problematic regarding the plausibility of appearance mysterianism.

I believe that this is a mistake. I agree that the support the apparent mysteriousness of consciousness provides to the idea of mysteriousness of consciousness might be defeated without being undercut. More specifically, it might be rebutted without being undercut. However, I also hold that such a defeat would not render appearance mysterianism stable. So, without answering the illusion question and thereby undercutting the support in question, the stability challenge cannot be adequately met: the defeat in question must be of a particular, viz. undercutting, kind.

Now, rebutting defeat occurs when a subject who has evidence that $p$ acquires an extra piece of evidence that not-p. Here is a case. Suppose that, as in the previous, undercutting case, I enter my friend's reading room and have an experience as of seeing some red books. Suppose further that in this case, my trustworthy friend, with 
an authoritative tone of voice, tells me that there are no red books in the room (and perhaps adds that it merely appears that there are). In this case, and unlike in the previous case, my friend's testimony is evidence that there are no red books, while my experience is evidence that there are. The two combined, it seems that my total evidence does not support the belief that there are red books (or the belief that there are no red books). In this case, the support my experience provides to the belief that there are red books is rebutted by my friend's testimony. But it is not undercut because even after my friend's testimony, my experience still continues to support the belief that there are red books.

So, the support the apparent mysteriousness of consciousness provides to the idea of mysteriousness of consciousness might be rebutted by considerations in favor of the idea of non-mysteriousness of consciousness. One such consideration, for instance, is known as the causal argument. The argument starts with the premise that the physical world is "causally closed" in the sense that every physical effect has a sufficient physical cause. It continues with the assertion that mental (conscious) events have physical effects (e.g., that I feel thirsty causes my reaching the bottle). The conclusion is that conscious events must be physical events (assuming that there is no causal over-determination). The causal argument is an argument in favor of the idea of the non-mysteriousness of consciousness and thus rebuts the support the apparent mysteriousness of consciousness provides to the idea of mysteriousness of consciousness.

However, rebutting defeat is a double-edged sword: the support provided to $p$ by $E$ is rebutted by $E^{\prime}$ just in case the support provided to not- $p$ by $E^{\prime}$ is rebutted by $E$. So, if the support the apparent mysteriousness of consciousness provides to the idea of mysteriousness of consciousness is rebutted by the causal argument, then the support the causal argument provides to the idea of non-mysteriousness of consciousness is also rebutted by the apparent mysteriousness of consciousness. This means that appearance mysterianism can hardly find relief in the fact that the causal argument rebuts the support the apparent mysteriousness of consciousness provides to the idea of mysteriousness of consciousness. This is because the rational attitude on that very fact taken by itself is not to believe that the mystery of 
consciousness is merely apparent but to suspend judgment regarding whether it is merely apparent or real.

The overall lesson is that in order to meet the stability challenge, the defeat in question must be of the undercutting kind and that in turn can be provided by (and plausibly, only by) answering the illusion question. So, the stability challenge cannot be met without answering the illusion question. An adequate answer to the illusion question does not only automatically qualify as a proper part of an adequate response to the stability challenge but it is also required for such a response. Let us then examine some answers provided by the appearance mysterians to the illusion question.

\section{The Illusion Question}

An adequate answer to the illusion question must meet three conditions at once: it must account for the depth of the (so-called) merely apparent mystery without abandoning the idea that it is apparent after all and also without replacing that apparent mystery by some other, equally challenging (apparent) mystery. The danger awaiting the appearance mysterian here is that if the apparent mysteriousness of consciousness proves to be resistant to be tamed by the physical, then the temptation to appeal to something really mysterious or to bring in some novel apparent mysteries grows higher, a temptation which one might unsuspectingly yield to. The more resistant to a non-mysterious explanation the apparent mysteriousness is, the bigger the temptation to account for it by an appeal to a real mystery will be.

Among the three conditions mentioned, what I will, for obvious reasons, call "the no-abandonment condition" and "the no-replacement condition" are selfexplanatory; and, let me make a couple of remarks about the third one, what I will call "the depth condition". How deep is the apparent mysteriousness of consciousness? How strongly resistant does consciousness appear to be to an explanation in terms of bodily features? How bleak does the future for our attempts to give a physical explanation of consciousness look? It certainly appears to us that consciousness is mysterious; and the question about the depth of the appearance is a question about 
whether the appearance itself appears temporary or permanent. Does it look as if there is nothing that blocks in principle a physical explanation of consciousness? Or, does it look as if our current lack of a physical explanation is a symptom of something that runs deeper?

I take it that the apparent mysteriousness of consciousness is as deep as the apparent mysteriousness of any given phenomenon can get. It is not merely that we currently have no physical explanation of consciousness but also that we don't even have the beginnings, however rough and rudimentary they might be, of such an explanation. Even more depressingly, we don't have a clue about how to make a start on this front that at least has a glimmer of promise. It is true that we know more, much more, about the brain than we did, say, a hundred years ago; however, the central philosophical question also keeps standing as perplexing as it was a hundred years ago: what does this (physical event) matter have to do with that (conscious event)? So, I take it that what the appearance mysterian needs to account for is not merely why consciousness appears to be currently unaccountable in physical terms but also, and more substantially, why it appears to be unaccountable in principle in such terms (that is, unaccountable in physical terms that we can possibly master).

Let me now move on to assessing three different answers offered by the appearance mysterians to the illusion question. One answer is that the reason why consciousness appears mysterious even if it is not mysterious is that we don't yet know enough the brain. According to this answer (which I shall call "the ignorance answer"), the apparent mysteriousness of consciousness is temporary, and there is no good reason to think that it will not vanish when we learn more about the workings of the brain. ${ }^{1}$ The ignorance answer has the potential to explain why consciousness appears to be currently unaccountable in physical terms; however, it does not explain why it appears to be unaccountable in principle in such terms. In fact, it simply misjudges and does not take seriously the depth of the apparent mysteriousness of consciousness and thus fails to appreciate what needs to be explained. It is trivially true that if we attain an explanation of consciousness in physical terms in the future, then the apparent mysteriousness of consciousness will vanish; however, this does

\footnotetext{
${ }^{1}$ The ignorance answer is defended most notably by Daniel Dennett (1992).
} 
not explain why it now appears to us that such an explanation of consciousness is in principle unattainable. The ignorance answer does not satisfy the depth condition and thus is inadequate.

A different and more popular answer among appearance mysterians to the illusion question takes the apparent mysteriousness of consciousness seriously. According to this answer, the reason why it now appears to us that a physical explanation of consciousness is in principle unattainable is due to the fundamentally different way we conceive and conceptualize some of the physical states of our brains. There are no non-physical (or non-functional) states of the brain, so a given state of a brain is as susceptible to an explanation as any physical state we can observe in the nature is. However, the answer goes, we conceptualize some of those brain states in a rather peculiar way, phenomenally; and, what explains why a physical explanation of consciousness is in principle unattainable is that phenomenal conceptualizations of those brain states are what we appeal to when we talk about consciousness and also that phenomenal conceptualizations are fundamentally disconnected from physical conceptualizations of those states. A phenomenal conceptualization of a brain state has no a priori links to its physical conceptualization, and it is therefore no wonder, according to this answer, that we balk at and feel perplexed by the question "how can we explain this (conceived phenomenally) in terms of this (conceived physically)?" However, consciousness is simply a constellation of physical brain states, albeit conceived phenomenally, and it is capable of receiving as much physical explanation just as any other. Let us call this answer to the illusion question "the phenomenal answer". ${ }^{2}$

The phenomenal answer evidently meets the depth condition: it accounts for why it appears to us that a physical explanation of consciousness is in principle unattainable. The way we conceptualize some physical states is what is responsible for the appearance in question. Given our phenomenal conceptualization of those states, the mysterious appearance is bound to occur. Furthermore, the phenomenal answer meets the no-abandonment condition: the mysteriousness of consciousness

\footnotetext{
${ }^{2}$ What I call "the phenomenal answer" is famously called "the phenomenal concept strategy" by Daniel Stoljar (2005). Some of its notable defenders are Brian Loar (1997), Christopher Hill (1997), and Katalin Balog (2012).
} 
is merely apparent according to this answer because consciousness is physical. However, it fails to meet the no-replacement condition: the phenomenal answer accounts for the apparent mysteriousness of consciousness by an appeal to our phenomenal conceptualization; however, that we have such a peculiar conceptualization of some physical brain states but not the others itself appears to be mysterious. Why do we conceptualize some physical brain states phenomenally while others are not susceptible to such a conceptualization? There seems to be nothing special in those physical states that we conceptualize phenomenally, no special physical feature that distinguishes them from the rest that we don't conceptualize phenomenally. So, on the phenomenal answer, the apparent mysteriousness of consciousness is explained only at the cost of creating a novel mysterious appearance, viz. the apparent mystery that we conceptualize only some physical brain states phenomenally while it seems that there is no relevant difference between those brain states and the rest which can explain this conceptualization feat.

The final answer I will consider to the illusion question agrees with the phenomenal answer that something more fundamental than a mere appeal to our ignorance is required to explain the apparent mysteriousness of consciousness. According to this answer, however, the reason why it appears to us that a physical explanation of consciousness is in principle unattainable has ultimately to do with a peculiar feature of our brains (rather than with a peculiar feature of our conceptualizations). The main intuition guiding this answer is that the acknowledgment that there must be something about the brain, a certain property of the brain, that explains why and how this (brain state) gives rise to that (conscious state) requires that that property be radically different from the other properties of the brain that we know and as we know them. This is because ordinary physical properties don't appear to be capable of rising up to the challenge of giving such an explanation. Ordinary physical properties of the brain aren't just cut out for the job; and, according to the thesis at hand, which I will call "the extra-property answer", we need to posit something extraordinary, an extraordinary property of the brain for an extraordinary explanatory mission, viz. that of explaining the fact that we are conscious. ${ }^{3}$ The extraordinary property in question

\footnotetext{
${ }^{3}$ The extra-property answer is defended most notably by Colin McGinn (1989).
} 
must satisfy two desiderata: it must be something radically different from other properties of the brain, so different in a way that makes it capable of explaining consciousness, while still being a property of the brain.

The extra-property answer to the illusion question satisfies the depth condition: if the extra-property answer is right, then the reason why it appears to us that a physical explanation of consciousness is in principle unattainable is that such an explanation must make reference to the extraordinary property of the brain and, given its extraordinary nature, that property might well fall beyond our cognitive reach. However, it fails to meet the no-abandonment condition: the extra-property answer explains the apparent mysteriousness of consciousness by an appeal to there being an extraordinary property of the brain, a property that differs radically from ordinary properties of the brain. The extraordinary property in question is simply a real (and not merely apparent) mystery built right into the heart of nature: to acknowledge that there is such an extraordinary property is to acknowledge that there is a real mystery. The extra-property answer invokes something really mysterious to account for the apparent mysteriousness of consciousness, a move that in effect commits that answer to treating, at one remove, consciousness itself as a real mystery.

We have assessed three different answers to the illusion question and found them wanting. Absent any other answers currently available, it is fair to draw the conclusion that consciousness is a real mystery, however unbelievable that might sound.

\section{References}

Balog, K. (2012). "In Defense of the Phenomenal Concept Strategy", Philosophy and Phenomenological Research 84, 1-23.

Dennett, D. (1992). Consciousness Explained. Back Bay Books.

Hill, C. (1997). "Imaginability, Conceivability, Possibility, and the Mind-Body Problem", Philosophical Studies 87, 61-85.

Loar, B. (1997). "Phenomenal States", in Ned Block, Owen Flanagan \& Güven Güzeldere (eds.), The Nature of Consciousness: Philosophical Debates, MIT Press.

McGinn, C. (1989). "Can we Solve the Mind-Body Problem?", Mind 98, 349-366.

Stoljar, D. (2005). "Physicalism and Phenomenal Concepts", Mind and Language 20, 469-494. 